\title{
Depressed type of inflammatory fibroid polyp of the colon
}

\author{
Kazuya Iwamoto • Masanori Sakashita • \\ Takuya Takahashi $\cdot$ Daisuke Obata $\cdot$ Shinwa Tanaka $\cdot$ \\ Masatoshi Fujii • Yoshinori Okabayashi
}

\begin{abstract}
Accepted: 8 June 2006 / Published online: 28 June 2006
(C) Springer-Verlag 2006
\end{abstract}

\section{Dear Editor:}

A 45-year-old man, who has been suffering from recurrent diarrhea and constipation, was referred to our hospital. Colonoscopy showed a reddened depressed lesion with marginal elevation in the transverse colon, near the hepatic flexure. After the mucosal surface was sprayed with indigo carmine or crystal violet dye, magnifying endoscopy revealed a type VI pit pattern of the Kudo's classification in the central depression and a type I pit pattern in the surrounding area. Endoscopic ultrasonography showed that the lesion was infiltrating into the submucosal layer. Therefore, we considered that the lesion might be a submucosal invasive colon cancer presenting like a submucosal tumor, although the biopsy specimen taken from the lesion did not contain distinct malignant components. Since we could not deny that the lesion was a malignant tumor, laparoscopy-assisted right hemicolectomy was performed. The resected specimen measured $18 \times 18 \mathrm{~mm}$ in size. Zoom stereomicroscopy of the resected specimen demonstrated a type $\mathrm{VN}_{\mathrm{N}}$ pit pattern in the part of the central depression, a type VI pit pattern in the rest area of the central depression, and a type I pattern in the surrounded area. Histological examination showed an enormous proliferation in the connective tissues and infiltration of eosinophils mainly in the submucosa. There were thick-

K. Iwamoto $(\bowtie) \cdot$ D. Obata $\cdot$ S. Tanaka $\cdot$ Y. Okabayashi

Department of Internal Medicine, Kobe Red Cross Hospital,

1-3-1 Wakinohamakigandorri, Chuo-ku,

651-0073 Kobe, Japan

e-mail: iwamotok@r3.dion.ne.jp

M. Sakashita $\cdot$ M. Fujii

Department of Gastroenterology, Kobe Red Cross Hospital,

Kobe, Japan

T. Takahashi

Department of Pathology, Kobe Red Cross Hospital,

Kobe, Japan walled vessels surrounded by the proliferative fibromuscular fibers, resulting in onion skin appearances. These findings were compatible with inflammatory fibroid polyp.

The term "inflammatory fibroid polyp" was introduced by Helwig and Rainer in 1953. Its etiology, suggested to be the involvement of inflammatory mechanisms or reactive responses, remains unclear. It usually forms a pedunculated or subpedunculated submucosal tumor, with erosion or ulceration overlying the mucosa. The lesions are solitary and occur predominantly in the stomach, small intestine, and rarely in the colon. Because it is usually located in the base mucosa and submucosa, difficulty in obtaining a histological diagnosis before treatment was reported.

Magnifying colonoscopy with chromoendoscopy was reported to be useful in differentiating almost all lesions detected at colonoscopy before histological evaluation. Kudo proposed the classification of the pit patterns: types I, II, IIIL, IIIs, IV, and V. Type V is divided into two types: VI and VN. In this classification, type I corresponds to normal gland, whereas type $\mathrm{V}$ corresponds to cancerous gland with type $\mathrm{VN}_{\mathrm{N}}$ pointing towards submucosal infiltration.

In the present study, magnifying endoscopy showed a type VI pit pattern in the central depression and a type I pattern in the surrounding area. These findings, as well as depressed type macroscopic appearance, suggested that the lesion might be a depressed colon cancer, which is thought to posses high malignant potential. Zoom stereomicroscopic findings corresponded to the endoscopic findings, except for a demonstration of type $\mathrm{VN}_{\mathrm{N}}$ pit pattern in the part of the central depression. In this case, the area showing type $\mathrm{VN}_{\mathrm{N}}$ or VI pit pattern is thought to correspond to the lesion where the surface epithelium was abraded by benign erosive inflammation.

With this result, it is suggested that endoscopists should be aware, when performing endoscopic examinations, that a colonic depressed lesion presenting a type $\mathrm{V}$ pit pattern can be an inflammatory fibroid polyp. 